\title{
DESIGN AND IN VITRO RELEASE KINETICS OF LIPOSOMAL FORMULATION OF ACYCLOVIR
}

\author{
DEBATRI ROY, SUDIPTA DAS*, ARNAB SAMANTA
}

PG Research Laboratory, Department of Pharmaceutics, Netaji Subhas Chandra Bose Institute of Pharmacy, Chakdaha, Nadia, West Bengal, India

Email: sudiptapharmacy6@gmail.com

Received: 14 Jul 2019, Revised and Accepted: 02 Sep 2019

\section{ABSTRACT}

Objective: The objective of this study was to formulate and evaluate liposomes loaded with acyclovir.

Methods: Liposomal formulation of acyclovir was prepared by "thin lipid film method". Mixture of phosphotidyl choline (soya lecithin), acyclovir and cholesterol of varying weight ratio was used for the preparation of liposomes. Total nine batches were prepared and each batch was prepared in triplicate. All batches were evaluated by drug entrapment and release kinetic studies. Stability study was carried out with $\mathrm{F}_{4}$ batch.

Results: $\mathrm{F}_{2}$ and $\mathrm{F}_{9}$ formulations showed better drug entrapment efficiency compared to the other batches. The drug entrapment efficiency of all batches was found within the range of $51.42 \%$ to $79.0 \% \mathrm{~F}_{4}$ shows highest release of about $79.0 \%$ in $4 \mathrm{~h}$. $\mathrm{F}_{4}$ formulation was found to follow significantly zero-order release kinetics. Short term stability study of formulation $\mathrm{F}_{4}$ showed no significant change in release kinetics.

Conclusion: The $\mathrm{F}_{4}$ batches showed promising results compared to other formulations. No changes were founded during the short-term stability study of $\mathrm{F}_{4}$.

Keywords: Liposomes, Acyclovir, Phosphatidylcholine, Cholesterol, Release kinetics

(C) 2019 The Authors. Published by Innovare Academic Sciences Pvt Ltd. This is an open-access article under the CC BY license (http://creativecommons.org/licenses/by/4.0/) DOI: http://dx.doi.org/10.22159/ijap.2019v11i6.34917

\section{INTRODUCTION}

Liposomes are composed of a lipid bilayer which used as drug delivery vehicles. The hydrophobic chains of the lipids form the bilayer and the polar head groups of the lipids are towards the extra vesicular solution and inner cavity. Liposomes have polar and non-polar groups on the same molecule. Due to hydrophilic and lipophilic interactions they can form self-organized and ordered structures in solvents [1]. Liposomes can give ligand targeted, long-acting and triggered release systems. Liposome preparation techniques may be broadly classified into two typesbulk method and film method. In bulk method phospholipids are transferred from an organic phase into an aqueous phase. In the film method lipid films are deposited on a substrate followed by hydration to get the liposomes. Liposomal preparations have also been classified according to mean size, polydispersity and lamellarity of liposomes [2]. The hydrophilic part of liposomes is mainly phosphoric acid bound to water-soluble molecules. On the other hand hydrophobic part of liposome contains two fatty acid chains with 10 to 24 carbon atoms and each chain contains 0 to 6 double bonds [3].

Most antiviral agents which have activity against the herpes group of viruses belong to a class of compounds known as nucleosides. Acyclovir or 9-(2-hydroxyethoxy methyl) guanine is one of those antiviral agents [4]. Oral absorption of acyclovir is about 20\% [5, 6]. Serious infections must be treated with intravenous acyclovir. The drug distributes all over the body. Cerebrospinal fluid (CSF) concentration is less than half of plasma concentration. The drug is excreted unchanged through kidneys. Excretion can be by both glomerular filtration and tubular secretion. Acyclovir has a halflife of about 2.5 to $3 \mathrm{~h}$ that can increase in renal impairment. Most importantly acyclovir used in encephalitis caused by herpes simplex, skin infections with herpes simplex, including labial and genital herpes can be treated by topically applied acyclovir $[7,8]$. Acyclovir has different routes of administration like topical, oral and intravenous but it is discovered that intravenous formulation has most profound antiviral effect in case of in vivo study [9] Intramuscular or subcutaneous administration of liposomal formulation in suspension form can increase the therapeutic efficacy of the encapsulated drug. When liposomal formulation was administered intramuscularly, toxicity of therapeutic drugs was found to be reduced [9-11]. Liposomes can be used as delivery vehicles for chemotherapeutics [12]. Human Immunodeficiency Virus (HIV)-infected persons have more risks of herpes-zoster than non-HIV patients. In those cases acyclovir can reduce incidence in HIV-infected persons [13].

\section{MATERIALS AND METHODS}

\section{Materials}

Acyclovir, Phosphatidyl Choline (soya lecithin), and cholesterol were purchased from Yarrow Chem, Mumbai. Chloroform was purchased from Merck, India. All the other chemicals and reagents used were of analytical grade.

\section{Preparation of liposomes}

For the preparation of acyclovir liposomes "Thin Lipid Film Method" was employed. A mixture of phosphatidylcholine (soya lecithin), acyclovir and cholesterol of specific ratio were dissolved in $5 \mathrm{ml}$ of chloroform [14-16] with sonication for 10 $20 \mathrm{~min}$. The organic solution was taken in a round bottom flask and rotated on a thermostatic water bath $\left(30{ }^{\circ} \mathrm{C}-35^{\circ} \mathrm{C}\right)$ to evaporate the organic solvent [17]. The drying process was continued for about $40 \mathrm{~min}$. The mixture of lipid and drug was deposited as a thin film in the round-bottom flask after complete evaporation of chloroform [18]. Proper drying plays a very important role in the preparation of liposomes [19]. After drying, $5 \mathrm{ml}$ phosphate buffer ( $\mathrm{pH} \mathrm{7.4)}$ was added and sonication was applied until a milky white suspension was formed (temperature maintained at $35^{\circ} \mathrm{C}$ ). Liposomes were formed due to spontaneous hydration of lipids in aqueous media. The suspension was allowed to stand for overnight. Sonication for 1 hour was applied to reduce the particle size [20].

\section{Entrapment study}

Liposomal suspension, $1 \mathrm{ml}$ was taken in a glass centrifuge tube, followed by centrifugation [21] at $3500 \mathrm{rpm}$ for $40 \mathrm{~min}$. The supernatant was completely removed by a micropipette followed by washing the pellet with $5 \mathrm{ml}$ of phosphate buffer solution ( $\mathrm{pH} 7.4$ ) to remove any un-entrapped drug. Both of the solutions were pooled and the clear supernatant solution was diluted. The absorbance was measured in UV-spectrophotometer (ELICO SA165) at $253 \mathrm{~nm}$ against phosphate buffer (pH 7.4) as blank. 


\section{In vitro drug release study}

The drug release study of acyclovir from different liposomal formulations was carried out by diffusion technique [22]. Liposomal acyclovir suspension, $5 \mathrm{ml}$ was taken in a dialysis bag [Abron Visking tube BE-253-b] $[23,24]$. Both ends of the bag were clipped. The bag was immersed in a medium of phosphate buffer (pH 7.4). The medium was maintained at $37 \pm 0.2^{\circ} \mathrm{C}$ with constant stirring by a magnetic stirrer. Aliquots of samples $(2 \mathrm{ml})$ of the medium were withdrawn at every $15 \mathrm{~min}$ interval up to $4 \mathrm{~h}$. The volume of the medium was made up with $2 \mathrm{ml}$ pre-warmed phosphate buffer ( $\mathrm{pH}$ 7.4) $[22,23]$ after each sampling. The concentration of the sample was determined by taking the absorbance in UV-spectrophotometer (Elico SA165) at $253 \mathrm{~nm}$ against phosphate buffer (pH 7.4) as blank.

\section{Drug stability study}

Freshly prepared liposomal formulation of acyclovir that gave a better-sustained release pattern was stored in Eppendrof's tube at 4
${ }^{\circ} \mathrm{C}$ [25] for one month. At the end of 1 mo, samples were analyzed by drug release study.

\section{RESULTS}

\section{Drug entrapment efficiency}

The un-entrapped drug was extracted out from the liposomal suspension with the help of phosphate buffer $(\mathrm{pH} 7.4)$ and the amount of un-entrapped drug was determined. The amount of drug entrapped was determined by subtracting from the amount of drug taken initially. Entrapment efficiency was determined from the following equation [26]:

Drug entrapment efficiency $=\frac{\text { Actual drug content in liposomal acyclovir }}{\text { Theoretical drug content in liposomal acyclovir }} \times 100$

The Drug Entrapment Efficiency (\%) values found with various cholesterol proportions (mol \%) are shown in fig. 1.

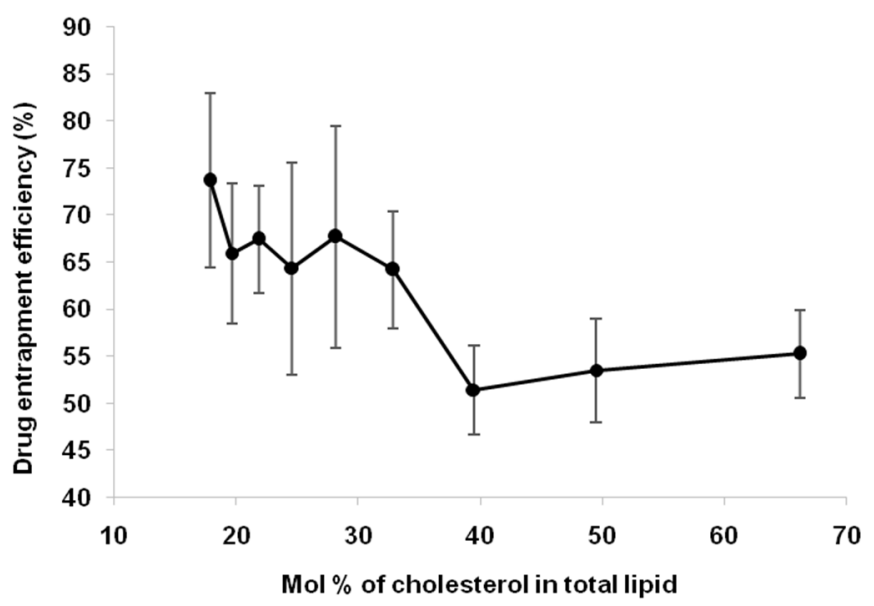

Fig. 1: Drug entrapment efficiency (\%) versus mol \% of the cholesterol in total lipid. ( $N=3$, Mean \pm SD)

From fig. 1 it is evident that \% entrapment increases with a decrease in mol \% of the cholesterol in the liposome and it became lowest at $39.5 \mathrm{~mol} \%$ cholesterol $\left(\mathrm{F}_{3}\right)$, the lowest entrapment efficiency being $51.42 \%$. The highest entrapment efficiency (73.6\%) was found with $\mathrm{F}_{9}$ having cholesterol of $17.9 \mathrm{~mol} \%$.

\section{In vitro drug release study from liposomes}

The dissolved acyclovir was diffused from liposome to the suspending medium and then permeated [27] across the dialysis membrane. Passage of the drug across dialysis membrane is a passive process. Therefore the rate of permeation was assumed to be higher than the rate of release of acyclovir from the liposome in the suspending medium. The percentage of drug released from the liposome was plotted against time and the release profiles of all the liposomal formulations are plotted in fig. $2 . \mathrm{F}_{4}$ shows highest release of about $79.0 \%$ in $4 \mathrm{~h}$.

\section{Determination of $\mathrm{t}_{30}$ (Time required for $30 \%$ drug release)}

In order to compare between the release profiles of various formulations a parameter, time required for $30 \%$ release $\left(\mathrm{t}_{30}\right)$, was determined. The release rate was found to be highest in $F_{4}$ containing $32.9 \mathrm{~mol} \%$ of cholesterol in the lipid phase which is exhibited by the lowest $t_{30}$ value in fig. 3 . The corresponding $t_{30}$ value of $\mathrm{F}_{4}$ was found to be $97.5 \mathrm{~min}$.

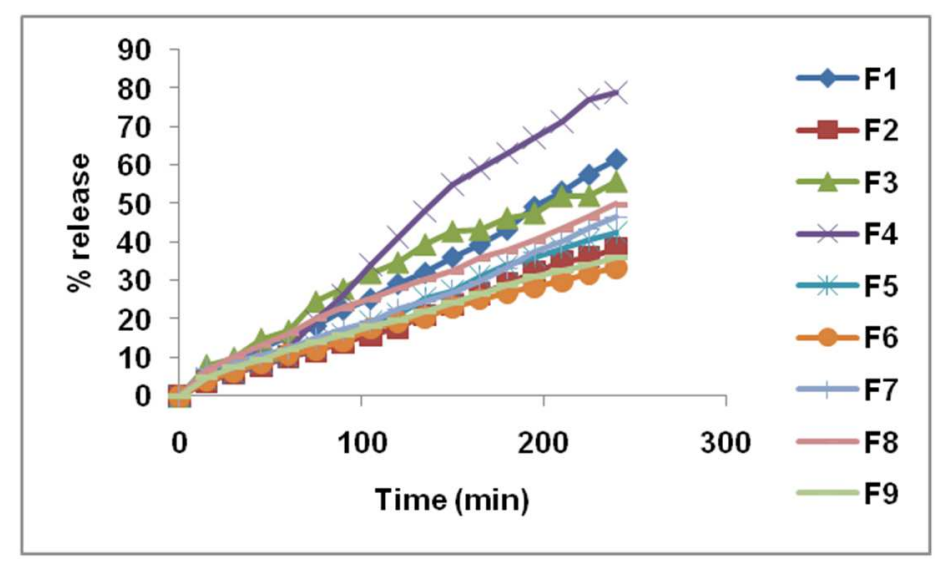

Fig. 2: Drug release study of $F_{1}, F_{2}, F_{3}, F_{4}, F_{5}, F_{6}, F_{7}, F_{8}$ and $F_{9}$ 


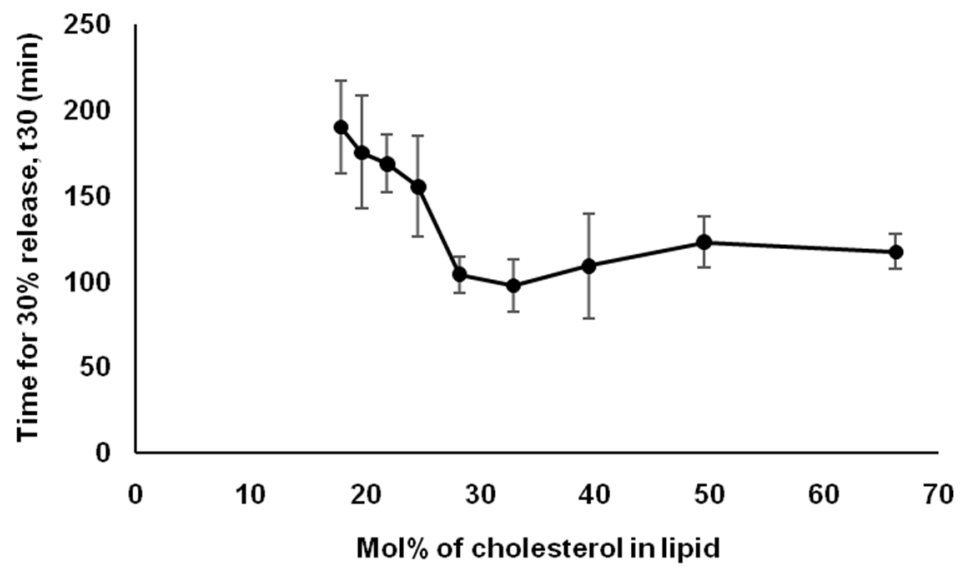

Fig. 3: Time taken for $30 \%$ release of drug from all formulations, $(\mathrm{N}=3, \mathrm{Mean} \pm \mathrm{SD})$

\section{In vitro drug release kinetic modeling}

The dissolved acyclovir was diffused from liposome to the suspending medium and then permeated [27] across the dialysis membrane. Passage of the drug across dialysis membrane is a passive process. Therefore the rate of permeation was assumed to be higher than the rate of release of acyclovir from the liposome in the suspending medium. In order to model the release kinetics of various liposomal formulations, the release profiles of drug from the liposomes were fitted to Zero-order, First-order, Higuchi, HixonCrowell, and Korsmeyer-Peppasequations. The correlation coefficients obtained from each model is displayed in table 1.

Table 1: Determination of coefficient of correlations of various kinetic models

\begin{tabular}{llllll}
\hline Formulation & Zero-order & First-order & Higuchi & Hixon-crowell & Korsmeyer-peppas \\
\hline F1 & $0.9923 \pm$ & $0.9886 \pm$ & $0.9176 \pm$ & 0.9916 & 0.8435 \\
& 0.0088 & 0.0113 & 0.0482 & \pm 0.0066 & \pm 0.0520 \\
F2 & $0.9893 \pm$ & 0.9815 & 0.9729 & 0.9839 & 0.8025 \\
& 0.0164 & \pm 0.0196 & \pm 0.0052 & \pm 0.0187 & \pm 0.0499 \\
F3 & $0.9624 \pm$ & $0.9715 \pm$ & $0.9711 \pm$ & $0.9704 \pm$ & $0.9026 \pm$ \\
F4 & 0.0417 & 0.0237 & 0.0061 & 0.0275 & 0.0565 \\
F5 & $0.9810 \pm$ & 0.9589 & 0.9649 & 0.9752 & 0.7481 \\
& 0.0171 & \pm 0.0142 & \pm 0.0066 & \pm 0.0076 & \pm 0.0390 \\
F6 & 0.9954 & 0.9888 & 0.9735 & 0.9730 & 0.8564 \\
F7 & \pm 0.0038 & \pm 0.0132 & \pm 0.0058 & \pm 0.0104 & \pm 0.0992 \\
F8 & 0.9910 & 0.9642 & 0.9508 & 0.9803 & 0.7970 \\
F9 & \pm 0.0056 & \pm 0.0547 & \pm 0.0426 & \pm 0.0254 & \pm 0.1374 \\
& $0.9826 \pm$ & $0.9637 \pm$ & $0.9761 \pm$ & $0.9712 \pm$ & $0.1474 \pm$ \\
& 0.0214 & 0.0432 & 0.0128 & 0.0347 & 0.1522 \\
& $0.9923 \pm$ & $0.9886 \pm$ & $0.9176 \pm$ & $0.9916 \pm$ & $0.8435 \pm$ \\
\end{tabular}

The mean values from 27 formulations ( 9 formulations x triplicate) are plotted against various kinetics models for comparison (vide fig. 4). From fig. 4 it is evident that the highest $\mathrm{R}^{2}$ value was found with Zero-order release kinetics.

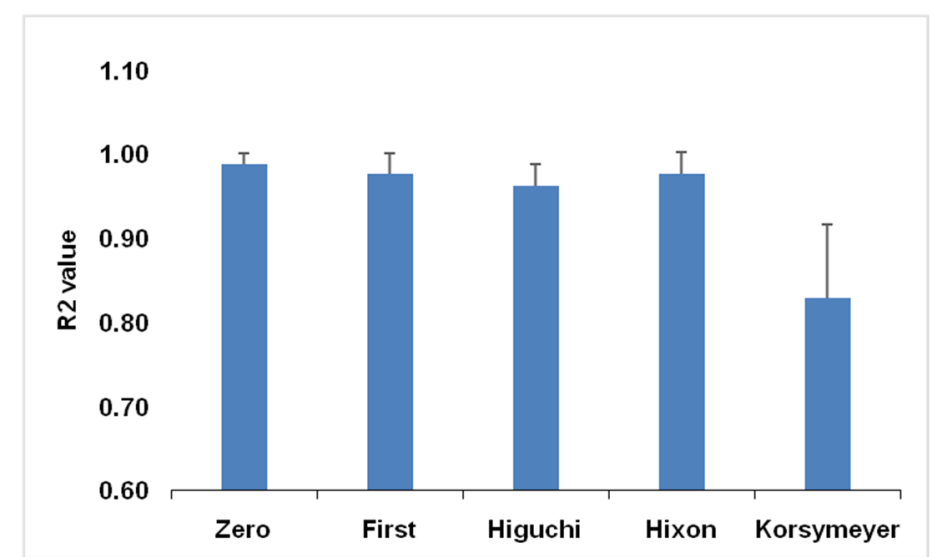

Fig. 4: The correlation coefficient values $\left(R^{2}\right)$ obtained from different kinetic models, (Mean $\left.\pm S D, n=27\right)$ 
The mean $\mathrm{R}^{2}$ values of Zero-order, First-order and Hixon-Crowell models were found to be close to each other. Hence, two-sample ttests between (i) Zero-order and First-order and (ii) Zero-order and Hixon-Crowell models were carried out with Minitab 18 software. In both cases, the difference between two means was found to be significant at $95 \%$ confidence level. Therefore, the $\mathrm{R}^{2}$-value of Zeroorder kinetics was found to be significantly highest among Zero, First and Hixon models. Therefore, it may be concluded that the release of acyclovir from the liposomes may be assumed to follow zero-order kinetics.

\section{Drug stability study}

Table 2: Drug stability study

\begin{tabular}{llllll}
\hline Time & Batch & Color & Percentage of drug entrapment efficiency & $\mathbf{t}_{\mathbf{3 0}}(\mathbf{m i n})$ & Changes in physical properties \\
\hline $1 \mathrm{mo}$ & $\mathrm{F}_{4}$ & Yellow & $62.38 \pm 5.24$ & $101.44 \pm 7.65$ & No changes \\
\hline
\end{tabular}

$(\mathrm{N}=3$, Mean $\pm \mathrm{SD})$

Formulation $\mathrm{F}_{4}$ was stored in three Eppendorf's tubes for one month. At the end of one month, samples were analyzed and the results are displayed in table- 2 . The values of drug entrapment efficiency and $t_{30}$ were found to be altered insignificantly. Therefore, the liposomes may be assumed to be stable till one month.

\section{DISCUSSION}

Liposomes function as drug carriers. Liposomes formation took place when phospholipids were dispersed in the aqueous medium and due to hydrophilic interaction of the lipid head groups with water. [28] In this study, total nine batches of liposomal acyclovir were prepared and each batch repeated thrice. The formulation contained several excipients like Lecithin, Cholesterol and Chloroform (solvent). Liposomal formulation of acyclovir was prepared by "Thin Lipid Film Method". In this method sonication was a vital step for mixing up of all excipient, including the drug. Lecithin is a very dense excipient to be suspended properly without sonication. After proper mixing of all ingredients it was very important to dry the suspension in water bath with rotational movement, which would help in the formation of thin lipid film. The evaporation process was continued until the smell of chloroform was present. The freshly prepared suspensions were kept for overnight at $4{ }^{\circ} \mathrm{C}$ in refrigerator before any evaluation procedure.

Drug entrapment study was performed and better entrapments were found from batch $F_{2}$ and $F_{9}$ which gave drug entrapment efficiency respectively about $75.49 \pm 0.51 \%$ and $73.637 \pm 9.26 \%$. Drug release study of all batches was performed by using phosphate buffer ( $\mathrm{pH}$ 7.4) medium. After release study it was found that formulation $\mathrm{F}_{4}$ showed higher percentage release $(79.021 \%)$ and $\mathrm{F}_{1}$ showed second-highest percentage release $(63.642 \%)$ in $4 \mathrm{~h}$. The release data was fitted to different kinetic models like Zero order, First order, Higuchi, Hixoncrowell and Korsmeyer-peppas. The highest correlation coefficients was found with zero-order model ( $r=0.9970)$ among all models. Hence, it may be concluded that the release of Acyclovir from liposomes follows zero-order kinetics and gave controlled drug release [29-31]. All nine batches followed zeroorder kinetics these means that they all lies under control release drug delivery system. According to the kinetic study $F_{9}$ followed zero-order kinetics rather than other batches because they showed higher correlation coefficient compared to other formulations. That means these formulations followed a controlled release drug delivery system. From fig. 3 it is evident that $\mathrm{F}_{4}$ (Cholesterol 32.9 mol\%) showed lowest $t_{30}$ value, in other words, the highest release rate among all other formulations. The lowest release rate corresponding to the highest $\mathrm{t}_{30}$ value was found from $\mathrm{F}_{1}$ formulation containing $66.2 \mathrm{~mol} \%$ of cholesterol. So it may be concluded that rate of release of Acyclovir took place with decreasing cholesterol mol\%. Therefore, by controlling the cholesterol mol\% the rate of release of acyclovir may be controlled from liposomal formulations. From the stability study results with $\mathrm{F}_{4}$ formulation the drug entrapment efficiency and the release rate (exhibited by $t_{30}$ value) changes were found after one month but those were insignificant.

\section{CONCLUSION}

The project was performed to exploit the activity of Acyclovir in the form of a liposome. Nine batches of a liposomal formulation of
Acyclovir with varying proportions of cholesterol and lecithin were prepared and evaluated to estimate their entrapment efficiency, release rate, drug release kinetics and stability testing. Most of the batches were found to follow zero-order release kinetics. $F_{3}$ and $F_{9}$ showed lowest and highest drug entrapment efficiencies, respectively with a pattern of increasing entrapment efficiency with decreasing proportion of cholesterol in the lipid phase. From the $\mathrm{t}_{30} \%$ drug release study it was founded that $\mathrm{F}_{4}$ gave slowest drug release and $\mathrm{F}_{9}$ gave the highest drug release rate. Therefore two or more formulations with varied release rates can be combined to produce a formulation with a required release rate. As $\mathrm{F}_{4}$ shows highest release pattern in $4 \mathrm{~h}$, the stability testing of this formulation was carried out and the formulation was found to be stable after one month's storage at $4{ }^{\circ} \mathrm{C}$ temperature. Though, long term stability study and clinical trial is required for future development of this dosage form.

\section{ACKNOWLEDGMENT}

The authors thank the authority of Netaji Subhas Chandra Bose Institute of Pharmacy, Chakdaha, West Bengal, India for providing the necessary facilities to perform the present study.

\section{AUTHORS CONTRIBUTIONS}

All authors contributed equally

\section{CONFLICT OF INTERESTS}

The authors declared no conflict of interest

\section{REFERENCES}

1. Lasic Dan D. Novel applications of liposomes. Trends Biotechnol 1998;16:307-21.

2. Patil Yogita P, Jadhav Sameer. Novel methods for liposome preparation. Chem Phys Lipids 2014;177:8-18.

3. Kulkarni PR, Yadav JD, Vaidya KA. Liposomes: a novel drug delivery system. Int J Curr Pharm Res 2011;3:10-8.

4. Schaeffer Howard J. Acyclovir chemistry and spectrum of activity. Am J Med 1982;73:4-6.

5. Tripathi K. Essentials of medical pharmacology. $5^{\text {th }}$ Ed. New Delhi: Jaypee brother's medical publishers (P) Ltd; 2008.

6. Mehta P. Acyclovir Pediatric Infectious Disease 2013;5:178-80.

7. King Dannie H. History, pharmacokinetics, and pharmacology of acyclovir. J Am Acad Dermatol 1988;18:176-9.

8. Keeney Ronald E, Wilson Sallie J. Acyclovir: a new era in antiviral chemotherapy. Clin Dermatol 1984;2:117-32.

9. Schwandt Nathan W, Mjos David P, Lubow Richard M. Acyclovir and the treatment of herpetic whitlow. Oral Surgery Oral Med Oral Pathol Oral Radiol 1987;64:255-8.

10. Tatode AA, Patil AT, Umekar JM, Telange RD. Investigation of effect of phospholipids on the physical and functional characterization of paclitaxel liposomes. Int J Pharm Pharm Sci 2017;9:141-6.

11. Sharma A, Sharma Uma S. Liposomes in drug delivery: progress and limitations. Int J Pharm 1997;154:123-40.

12. Liu Z, Bi Y, Sun Y Y, Hao F, Lu J, Meng Q, et al. Pharmacokinetics of a liposomal formulation of doxorubicin in rats. Saudi Pharm J 2017;25:531-6. 
13. Barnabas Ruanne V, Baeten Jared M, Lingappa Jairam R, Thomas Katherine $\mathrm{K}$, Hughes James $\mathrm{P}$, Mugo Nelly $\mathrm{R}$, et al. Acyclovir prophylaxis reduces the incidence of herpes zoster among HIV-infected individuals: results of a randomized clinical trial. J Infec Diseases 2015;213:551-5.

14. Sun W, Zhang N, Li A, Zou W, Xu W. Preparation and evaluation of N3-0-toluyl-fluorouracil-loaded liposomes. Int J Pharm 2008;353:243-50.

15. Singha Roy A, Das S, Samanta A. Design, formulation and evaluation of liposome containing Isoniazid. Int J Appl Pharm 2018;10:52-6.

16. Roy A, Das S, Samanta A. Diclofenac loaded cross-linked sodium alginate and gellan gum microspheres: development and in vitro release profile. World J Pharm Pharm Sci 2015;4:1718-29.

17. Zhang H. Thin-film hydration followed by the extrusion method for liposome preparation. Liposomes. Springer; 2017. p. 17-22.

18. Sinico C, Manconi M, Peppi M, Lai F, Valenti D, Fadda Anna M. Liposomes as carriers for dermal delivery of tretinoin: in vitro evaluation of drug permeation and vesicle-skin interaction. J Controlled Release 2005;103:123-36.

19. Dwivedi C, Verma S. Review on preparation and characterization of liposomes with the application. J Drug Delivery Ther 2014;4:116-29.

20. Moghimipour E, Salami A, Monjezi M. Formulation and evaluation of liposomes for transdermal delivery of celecoxib Jundishapur. J Natl Pharm Prod 2015;10:1-6.

21. Sunitha S, Thirupathi A, Vijaya K. Preparation and evaluation of liposome entrapped hydrogel complex system of itraconazole for enhanced transdermal permeation. J Pharm Sci Innovation 2014;3:25-9.

22. El-Gazayerly Omaima N, Hikal Ahmed H. Preparation and evaluation of acetazolamide liposomes as an ocular delivery system. Int J Pharm 1997;158:121-7.
23. Tsukamoto T, Hironaka K, Fujisawa T, Yamaguchi D, Tahara K, Tozuka Y, et al. Preparation of bromfenac-loaded liposomes modified with chitosan for ophthalmic drug delivery and evaluation of physicochemical properties and drug release profile. Asian J Pharm Sci 2013;8:104-9.

24. Nagarsenker MS, Londhe VY. Preparation and evaluation of a liposomal formulation of sodium cromoglicate. Int J Pharm 2003;251:49-56.

25. Guo Y, Shen L, Lu Y, Li H, Min K, Li L, et al. Preparation of rutinliposome drug delivery systems and evaluation on their in vitro antioxidant activity. Chin Herbal Med 2016;8:371-5.

26. Katsai OG, Ruban OA. Preparation and characterization of the liposomal delivery system of natural heme protein. Int J Appl Pharm 2019;11:718-25.

27. Haghiralsadat F, Amoabediny G, Helder MN, Naderinezhad S, Sheikhha $\mathrm{MH}$, Forouzanfar $\mathrm{T}$, et al. A comprehensive mathematical model of drug release kinetics from nanoliposomes, derived from optimization studies of cationic PEGylated liposomal doxorubicin formulations for drug-gene delivery. Artif Cells Nanomed Biotechnol 2018;46:169-77.

28. Vemuri S, Rhodes CT. Preparation and characterization of liposomes as therapeutic delivery systems: a review. Pharm Acta Helvetiae 1995;70:95-111.

29. Ramteke KH, Dighe PA, Kharat AR, Patil SV. Mathematical models of drug dissolution: a review. Scholars Acad J Pharm 2014;3:388-96.

30. Das S, Samanta A, Sekhar De H. Formulation, in vitro release kinetics and stability interaction of sustained-release tablets of metformin hydrochloride. Int J Pharm Pharm Sci 2015;7:41822.

31. Henriksen I, Sande SA, Smistad G, Agren T, Karlsen J. In vitro evaluation of drug release kinetics from liposomes by fractional dialysis. Int J Pharm 1995;119:231-8. 